\title{
ONE-DIMENSIONAL DIFFUSIONS
}

\section{AND THEIR EXIT SPACES*}

\author{
P. SALMINEN
}

\section{Introduction.}

Let $X=\left(\omega_{t}, t \geqq 0, P_{x}\right)$ be a one-dimensional regular canonical diffusion process with values in an interval $I=(a, b) \cong R$. Killing inside $I$ is not permitted but both boundary points $a$ and $b$ are assumed to be killing boundaries, i.e. a diffusion particle is living until it hits (if ever) the point $a$ or $b$. If a boundary point is hit, the particle is immediately transferred to a point $\Delta$ isolated from $I$. Consequently, the lifetime, denoted by $\zeta$, for this diffusion is simply

$$
\zeta=\inf \{t: \omega(t-)=a \text { or } b\} .
$$

A diffusion without killing in $I$ is fully characterized in $I$ by a continuous, strictly increasing scale function (unique up to linear transformations), denoted by $s$, and by a speed measure, denoted by $m$, with the property

$$
\forall \alpha, \beta: a<\alpha<\beta<b \quad 0<m\{[\alpha, \beta]\}<\infty .
$$

Further, we call a diffusion transient if

$$
\forall x \in I, A \text { compact } \in \boldsymbol{B}(I): P_{x}\left(\lambda_{A}<\infty\right)=1,
$$

where $\lambda_{A}=\sup \{t: \omega(t) \in A\},=0$ if the set in the brackets is empty, and $B(I)$ is the set of all Borel sets in $I$. Let $\tau_{\alpha}=\inf \{t: \omega(t)=\alpha\}$. Using the fact that for $a<\alpha<x<\beta<b$

$$
\begin{aligned}
P_{x}\left(\tau_{\beta}<\tau_{\alpha}\right) & =1-P_{x}\left(\tau_{\alpha}<\tau_{\beta}\right) \\
& =\frac{s(x)-s(\alpha)}{s(\beta)-s(\alpha)}
\end{aligned}
$$

it can be proved that (1.1) is equivalent to the following condition:

$$
s(a)>-\infty \text { and/or } s(b)<+\infty .
$$

Received August 23, 1982; in revised form February 17, 1983.

* The main part of the paper is included in the author's doctoral thesis presented at Abo Akademi in December 1981. 
Without loss of generality we shall only consider the case $s(a)=-\infty$ and $s(b)$ $<+\infty$. Then we have

$$
\forall x \in I: P_{x}\left(\lim _{t \rightarrow \zeta} \omega(t)=b\right)=1 ;
$$

and $a$ can be either entrance or natural, and $b$ either exit (not entrance) or natural or regular (see e.g. [6]).

The aim of this paper is to give a view to the Martin boundary theory and related things for the diffusion $X$ described above. Of course, the Martin compactification of the state space $I$ is trivial being just the closed interval $[a, b]$. However, it is seen that the minimal excessive transforms of $X$ have interesting properties. In our study we concentrate on these minimal transforms. Properties of general excessive transforms can be deduced from the minimals by integration.

In the next section we give the minimal excessive functions of $X$. The result is not suprising, and can be found in an implicit form, for example, in [3]. Our new probabilistic proof gives us, however, important and non-trivial information about the minimal excessive transforms of $X$.

In fact, it turns out that the distribution of the last exit time of the process $X$ from a fixed point $y \in I$ and the life-time distribution of the corresponding minimal transform coincide. This gives us a method to calculate the former distribution. The essential tool in our proof is the integral representation of excessive functions for the space-time diffusion $\left(t, X_{t}\right)$; the minimal functions of $X$ are expressed with aid of the minimal functions of $\left(t, X_{t}\right)$. Our easy and short proof highlights the usefulness of the analytical part of the Martin boundary theory.

The minimal transforms have clean and curious time reversal properties. Roughly speaking, a transform, which starts from $x$ and ends at $y$, is identical with a transform, which starts from $y$ and ends at $x$ but is run backwards in time. This was first, perhaps, noticed by Williams [12]. Since our result is given under the plain assumptiom (1.1) it is a slight generalization of Williams' result. The proof given here is straight forward compared with Williams' time change techniques. We also remark that this result can be proved using Nagasawa's general time reversal theorem (see [10]).

Further, an example involving Bessel diffusions in wide sense is discussed. In particular, the result of Barndorff-Nielsen et al. [1] about infinitely divisibility of the generalized inverse Gaussian distribution is established via time reversal.

In the final section of the paper we give a probabilistic, Markovian description of the minimal transform of $X$. For this we construct an "un-killed minimal transform". This process is then killed at the point $y$ "in an elastic manner" to produce the desired transform. 


\section{Minimal excessive functions.}

We start with

Proposition 1. For a given transient diffusion on $I=(a, b)$ with $s(a)=-\infty$ and $s(b)<+\infty$ the minimal excessive functions are

$$
\begin{aligned}
& k_{b}(x) \equiv 1, \\
& k_{a}(x)=s(b)-s(x)
\end{aligned}
$$

and

$$
k_{y}(x)= \begin{cases}1 & \text { if } x \leqq y, \\ \frac{s(b)-s(x)}{s(b)-s(y)} & \text { if } x \geqq y,\end{cases}
$$

where $x, y \in \boldsymbol{I}$.

Proof. The functions $k_{a}$ and $k_{b}$ are excessive because they are harmonic (see [3, Theorem 12.4]). Consequently, $k_{y}, y \in I$, are also excessive, because

$$
k_{y}=k_{b} \wedge \frac{1}{s(b)-s(y)} k_{a} \quad(f \wedge g:=\min (f, g)) .
$$

Further, $k_{b}$ is minimal because the diffusion converges to the boundary point $b$.

To prove that $k_{a}$ is minimal consider the $k_{a}$-transform of the diffusion. This is a regular diffusion with a transition function

$$
P^{k_{a}}(t ; x, A)=\int_{A} P(t ; x, d y) \frac{k_{a}(y)}{k_{a}(x)}, \quad A \in \boldsymbol{B}(I),
$$

where $P(t ; x, A)$ is the transition function of the diffusion $X$. We can calculate the scale function and the speed measure of the $k_{a}$-diffusion; these are

$$
s^{*}(x)=\frac{1}{s(b)-s(x)}
$$

and

$$
m^{*}(d x)=(s(b)-s(x))^{2} m(d x) .
$$

Clearly, because $s^{*}(a)=0$ and $s^{*}(b)=+\infty$, the $k_{a}$-diffusion converges to the point $a$; thus proving the minimality of $k_{a}$.

Next let us consider the functions $k_{y}$. We have

$$
\left.k_{y}(x)=P_{x} \text { (hit } y \text { before } b\right)=P_{x}\left(\lambda_{y}>0\right),
$$

where $\lambda_{y}=\sup \{t: \omega(t)=y\},=0$ if the set in the brackes is empty. 
Consequently (see [7]), the $k_{y}$-diffusion is identical in law with $X$ when conditioned to hit $y$ and killed at the last exit time from $y$. This proves that for all $x \in I P_{x}^{k_{y}}\left(\lim _{t \rightarrow \zeta} \omega(t)=y\right)=1$, implying the minimality of $k_{y}$.

Remark 2. The minimality of the functions $k_{y}$ is also easily proved by showing that $k_{y}$ has support at the point $y$. (For this concept see [2].)

Remark 3. It is known (see [5, Chapter 4.11]) that a regular diffusion has transition densities $p(t ; x, y)$ with respect to the speed measure:

$$
P_{x}(\omega(t) \in A)=P(t ; x, A)=\int_{A} p(t ; x, y) m(d y), \quad A \in \boldsymbol{B}(I) .
$$

The densities have the following properties:

(i) $p(t ; x, y),(\partial / \partial t) p(t ; x, y)$ are continuous on $(0, \infty) \times I \times I$.

(ii) $p(t ; x, y)=p(t ; y, x)>0$.

$p(t ; x, y)=\int p(t-v ; x, z) p(t ; z, y) m(d z), t>v>0, x, y \in I$.

(iii) For every fixed $x$ (or $y), p(t ; x, y)$ is a solution of the equation

$$
\frac{\partial}{\partial t} u(t, y)=\frac{d}{d m} \frac{d}{d s} u(t, y) .
$$

(iv) If $\boldsymbol{P}_{\boldsymbol{x}}(\zeta<\infty)=1$ for all $x \in \boldsymbol{I}$, then

$$
\begin{aligned}
\frac{\partial}{\partial t} P_{x}(\zeta<t) & =-\frac{d}{d s} p(t ; x, b) \\
& :=\lim _{\beta \uparrow b} \frac{p(t ; x, \beta)}{s(b)-s(\beta)} .
\end{aligned}
$$

Note that the symmetric density of the $k_{a}$-process is

$$
p^{k_{a}}(t ; x, y)=\frac{p(t ; x, y)}{(s(b)-s(x))(s(b)-s(y))},
$$

where $p(t ; x, y)$ is the symmetric density of the original diffusion.

\section{Last exit time distributions and time reversal properties of transient diffusions.}

It is clear from the proof above that if we start a $k_{y}$-diffusion from a point $x<y$ then the lifetime distribution of this process and the last exit-time distribution from the point $y$ of the original diffusion must coincide. So we make 
Proposition 4. Let $\zeta=\inf \{t: \omega(t) \notin I\}$; then

$$
P_{x}^{k_{y}}(\zeta \in d t)=\frac{p(t ; x, y)}{(s(b)-s(x)) \wedge(s(b)-s(y))} d t, \quad x, y \in I .
$$

Proof. Because the transition mechanism for the $k_{y}$-process is explicitly known, the distribution of $\zeta$ could be calculated directly using the facts given in Remark 3. We leave this, however to the reader, and choose a shorter way.

Firstly, because (see [5])

$$
\int_{0}^{\infty} p(t ; x, y) d t=(s(b)-s(x)) \wedge(s(b)-s(y))
$$

it is seen that the right hand side of (3.1) is a proper probability density.

Now let us consider the diffusion $X$ in space-time. Choose a point $(0, x)$ as a reference point (i.e. the point where $\left(t, X_{t}\right)$ is started; see also Remark 5). Then the Martin function (i.e. the minimal space-time excessive function) with support at a point $\left(t_{0}, \dot{y}_{0}\right)$ is simply

$$
(0, x) k_{\left(t_{0}, y_{0}\right)}(v, z)= \begin{cases}\frac{p\left(t_{0}-v ; z, y_{0}\right)}{p\left(t_{0} ; x, y_{0}\right)} & v<t_{0}, \\ 0, & \text { otherwise } .\end{cases}
$$

Let us assume that $x<y_{0}$, and insert on the line $y=y_{0}$ the density given in (3.1). According to the Martin boundary theory, if we integrate the minimal space-time excessive functions with support on this line against a probability measure living on the line we obtain a space-time excessive function $h$ such that $h(0, x)=1$ :

$$
\begin{aligned}
& h(v, z)=\int_{0}^{\infty}(0, x) k_{\left(t, y_{0}\right)}(v, z) \frac{p\left(t ; x, y_{0}\right)}{s(b)-s\left(y_{0}\right)} d t \\
& =\int_{s}^{\infty} p\left(t-v, z, y_{0}\right) \frac{1}{s(b)-s\left(y_{0}\right)} d t \\
& = \begin{cases}1, & \text { if } z \leqq y, \\
\frac{s(b)-s(z)}{s(b)-s\left(y_{0}\right)}, & \text { if } z>y_{0}\end{cases} \\
& =k_{y_{0}}(z) \text {. }
\end{aligned}
$$

It follows that the $h$-transform considered in the real line has the same finite dimensional distributions as the $k_{y_{0}}$-transform. Obviously, the case $x>y_{0}$ can be treated similarly, and the conclusion is the same. This completes the proof.

REMARK 5. For space-time processes a "correct" state space in the Martin 
boundary setting is $(0,+\infty) \times I$. This means that the process must be started from an isolated point behaving like $(0, x)$ and it arrives to the state space according to an entrance law. For Martin boundaries for some space-time processes see [9].

Corollary 6. Let $\lambda_{y}=\sup \{t: \omega(t)=y\},=0$ if the set in the brackets is empty. Then

(i) for $x \leqq y$ the distribution of $\lambda_{y}$ is proper and has the density

$$
P_{x}\left(\lambda_{y} \in d t\right)=\frac{p(t ; x, y)}{s(b)-s(y)} d t
$$

for $x>y$ the distribution of $\lambda_{y}$ has an atom at 0 , and the density of the continuous part is given by (3.2);

(ii) for $x \geqq y$

$$
P_{x}^{k_{a}}\left(\lambda_{y} \in d t\right)=\frac{p(t ; x, y)}{s(b)-s(x)} d t
$$

Proof. (i) follows directly from Proposition 4. To prove (ii) we apply (i) to the $k_{a}$-process to obtain for $x>y$

$$
P_{x}^{k_{a}}\left(\lambda_{y} \in d t\right)=\frac{p^{k_{a}}(t ; x, y)}{s^{*}(y)-s^{*}(a)}
$$

Using the formulae for $p^{k_{a}}$ and $s^{*}$, this can be written in the form (3.3).

Remark 7. J. Pitman and M. Yor have also recently (see [8]) obtained this result. Their proof lies, however, much deeper in the theory of diffusions than the one presented here.

The next proposition generalizes slightly the time-reversal theorem of D. Williams (see [12]):

Proposition 8. For all $x, y$ the processes

$$
\left\{\omega(\zeta-t), 0<t<\zeta, P_{x}^{k_{y}}\right\}
$$

and

$$
\left\{\omega(t), 0<t<\zeta, P_{y}^{k_{x}}\right\}
$$

are identical in law.

Proof. We shall prove, compare with the proof of Theorem 3.4 in [12], that 


$$
\begin{aligned}
& P_{y}^{k_{x}}\left(\omega(v) \in d z_{1}, \omega(v+t) \in d z_{2}, \zeta \in v+t+d u\right) d v \\
& \quad=P_{x}^{k_{y}}\left(\omega(u) \in d z_{2}, \omega(u+t) \in d z_{1}, \zeta \in u+t+d v\right) d u
\end{aligned}
$$

Using the Markov property this can be written

$$
\begin{aligned}
& P_{y}^{k_{x}}\left(\omega(v) \in d z_{1} ; P_{z_{1}}^{k_{x}}\left(\omega(t) \in d z_{2} ; P_{z_{2}}^{k_{x}}(\zeta \in d u)\right)\right) d \\
& \quad=P_{x}^{k_{y}}\left(\omega(u) \in d z_{2} ; P_{z_{2}}^{k_{y}}\left(\omega(t) \in d z_{1} ; P_{z_{1}}^{k_{y}}(\zeta \in d v)\right)\right) d u .
\end{aligned}
$$

Using Proposition 4 and the explicit expressions of the transition densities of the $k_{x}$ - and $k_{y}$-processes it is not difficult to see that both sides in (3.4) equal

$$
\frac{p\left(v ; y, z_{1}\right) p\left(t ; z_{1}, z_{2}\right) p\left(u ; z_{2}, x\right)}{(s(b)-s(x)) \wedge(s(b)-s(y))} m\left(d z_{1}\right) m\left(d z_{2}\right) .
$$

This completes the proof.

Remark 9.

(i) Of course, the essential fact needed to obtain the time-reversal property is, see (3.1),

$$
P_{y}^{k_{x}}(\zeta \in d t)=P_{x}^{k_{y}}(\zeta \in d t) .
$$

(ii) Taking the case $x<y$, Proposition 8 can be reformulated as follows: the processes

$$
\left\{\omega\left(\lambda_{y}-t\right), 0<t<\lambda_{y}, P_{x}\right\} \quad \text { and } \quad\left\{\omega(t), 0<t<\lambda_{x}, P_{y}^{k_{a}}\right\}
$$

are identical in law. If $P_{x}(\zeta<\infty)=1$ for all $x$ we can let $y \uparrow b$ above to obtain Theorem 2.5 in [12]. In this case $b$ will be an entrance, not-exit boundary point for the $k_{a}$-transform. Hence, it is possible to start this process from the point $b$.

(iii) Notice also that our Proposition 8 is valid in the stated form for all transient diffusion, i.e. for all cases $s(a)>-\infty$ and/or $s(b)<+\infty$.

We conclude this section by giving a few

Examples 10. (i) Let $X$ be a three dimensional Bessel process started from 0 and killed when it hits 1 . Here $s(x)=-1 / x$ and $m(d x)=2 x^{2} d x$, and 0 is an entrance, not-exit boundary point. Consider the $k_{0}$-transform, denoted $X^{0}$, of $X$ started from the point 1 . By Proposition $8, X^{0}$ and $X$ are timereversals of each other.

On the other hand, it is easily seen that a scale function for $X^{0}$ is $s^{*}(x)=$ $1 /(1-x)$ and a speed measure is $m^{*}(d x)=2(1-x)^{2} d x$. Consequently $1-X^{0}$ is also a three dimensional Bessel process started from 0 and killed when it hits 1 . 
This fact should be seen in the context of Brownian excursions, see, for example, [13, II 67].

(ii) Let $X$ be as above but let it run forever. In this case, the $k_{0}$-transform is a Brownian motion killed when it hits zero. Let $y>0$ be fixed and consider the $k_{y}$-transform of $X$. The equality (3.5) now takes the form

$$
P_{y}^{k_{0}}(\zeta \in d t)=P_{0}^{k_{y}}(\zeta \in d t) \text {. }
$$

But under $P_{.}^{k_{0}}$ the distribution of $\zeta$ is just the distribution of the first hitting time of zero by Brownian motion. Further under $P^{k_{y}}$ the distribution of $\zeta$ is just the distribution of the last exit time from $y$ by three-dimensional Bessel process. Consequently

$$
\begin{aligned}
P_{y}^{k_{0}}\left(\tau_{0} \in d t\right) & =P_{0}\left(\lambda_{y} \in d t\right) \\
& =\frac{p(t ; 0, y)}{-s(y)} d t \\
& =\frac{y}{\sqrt{2 \pi t^{3}}} \exp \left(-\frac{y^{2}}{2 t}\right) d t,
\end{aligned}
$$

because $s(x)=-1 / x$ and the symmetric transition density for $X$ (with respect to the speed measure $2 x^{2} d x$ ) is

$$
p(t ; 0, y)=\frac{1}{\sqrt{2 \pi t^{3}}} \exp \left(-\frac{y^{2}}{2 t}\right) .
$$

(iii) To give a more serious example consider a diffusion on $(0,+\infty)$ with the generator

$$
\boldsymbol{B}^{\alpha}=\frac{1}{2} \frac{d^{2}}{d x^{2}}+\left(\frac{1}{2 x}+\alpha \frac{I_{v}^{\prime}(\alpha x)}{I_{v}(\alpha x)}\right) \frac{d}{d x}, \quad \alpha>0,
$$

where $I_{v}$ is a modified Bessel function of the first kind and of the order $v \geqq 0$. If we let $\alpha \rightarrow 0$, we obtain a Bessel diffusion of the order $v$, i.e. a diffusion with the generator

$$
\boldsymbol{B}=\frac{1}{2} \frac{d^{2}}{d x^{2}}+\frac{2 v+1}{2 x} \frac{d}{d x}
$$

The three-dimensional Bessel process is a diffusion of this type with $v=0$.

Diffusions with the generator (3.6) were first introduced by S. Watanabe [11]. They also appear in [9], where it is shown that these are space-time excessive transforms of the usual Bessel diffusions, and could be called Bessel diffusions with a drift $\alpha$. 
From (3.6) we can calculate the scale function

$$
s(x) \equiv \int^{x} \frac{1}{I_{v}^{2}(\alpha y)} d y=-\frac{K_{v}(\alpha x)}{I_{v}(\alpha x)},
$$

where the equality is up to linear transformations and $K_{v}$ is a modified Bessel function of the second kind and of the order $v$. Further $s(0)=-\infty$ and $s(+\infty)$ $=0$. The generator of the $k_{0}$-process takes the form

$$
B^{-\alpha}=\frac{1}{2} \frac{d^{2}}{d x^{2}}+\left(\frac{1}{2 x}+\alpha \frac{K_{v}^{\prime}(\alpha x)}{K_{v}(\alpha x)}\right) \frac{d}{d x} .
$$

This is the diffusion used by Barndorff-Nielsen, Blæsild and Halgreen in their proof that the generalized inverse Gaussian distribution is infinitely divisible (see [1]). This distribution being the distribution of the first hitting time of 0 for (3.7) the infinitely divisibility is immediate.

The transition density for the diffusion (3.6) (see [11] on [9]) is known and so we can write down the last exit distributions. By a similar time reversal argument as in the example (ii) above, the result of Barndorff-Nielsen et al. follows immediately. For further results in this direction see [8].

\section{Markovian description of minimal transform.}

Let $y \in I$ be fixed, and consider the $k_{y}$-transform, denoted by $X^{y}$, of the diffusion $X$. Because $X^{y}$ is an excessive transform of $X$ and $X$ is strong Markov it follows that $X^{y}$ is also strong Markov. Further the paths of $X^{y}$ are continuous, and, hence, $X^{v}$ is a regular diffusion.

Let us introduce

$$
\begin{aligned}
m^{*}(d x) & =\left(k_{y}(x)\right)^{2} m(d x) \\
s^{*}(x) & = \begin{cases}s(x)-s(y), & x \leqq y, \\
(s(b)-s(y))^{2}\left(\frac{1}{s(b)-s(x)}-\frac{1}{s(b)-s(y)}\right), & x \geqq y .\end{cases}
\end{aligned}
$$

From the description of $X^{y}$ given in the proof of Proposition 1 it follows that $m^{*}$ and $s^{*}$ can be considered as a speed measure and a scale function for $X^{y}$. It is easily seen that the transition density

$$
q(t ; x, z)=\frac{p(t ; x, z)}{k_{y}(x)},
$$

for $X^{y}$ with respect to $m^{*}$ satisfies (i)-(iii) in Remark 3 .

Denote with $Z$ a conservative diffusion having the scale function $s^{*}$ and the speed measure $m^{*}$. Further let $L(t, x)$ be its local time normalized so that 


$$
E_{x}\left(\int_{0}^{\infty} e^{-\alpha t} d L_{t}(x)\right)=g_{\alpha}(x, x),
$$

where $g_{\alpha}$ is the Green function of $Z$.

Since $X^{y}$ is a diffusion with scale $s^{*}$ and speed $m^{*}$ we can construct it by killing $Z$ at the point $y$ in an appropriate manner. For this we have to calculate the killing measure of $X^{y}$. Let $a<\alpha<\beta<b$ and set

$$
\begin{aligned}
& p_{\alpha \beta}(x)=P_{x}^{k_{y}}\left(\tau_{\alpha}<\tau_{\beta}\right), \\
& p_{\beta \alpha}(x)=P_{x}^{k_{y}}\left(\tau_{\beta}<\tau_{\alpha}\right) .
\end{aligned}
$$

Then (see pp. 106-107 in [5]) on $(\alpha, \beta)$ the killing measure $x$ is given by

$$
\varkappa(A) \equiv \int_{A} \frac{p_{\alpha \beta}^{+}(d x)}{p_{\alpha \beta}(x)}=\int_{A} \frac{p_{\beta \alpha}^{+}(d x)}{p_{\beta \alpha}(x)},
$$

where $A$ is a Borel subset of $(\alpha, \beta)$,

$$
p^{+}(x)=\lim _{u \downarrow x} \frac{p(u)-p(x)}{s^{*}(u)-s^{*}(x)} \quad\left(p \equiv p_{\alpha \beta} \text { or } p_{\beta \alpha}\right)
$$

and $p^{+}(d x)$ is the Borel measure induced by the interval function $p^{+}(u, x]$ $=p^{+}(x)-p^{+}(u)(x>u)$. Let $\tau=\tau_{\alpha} \wedge \tau_{\beta} \wedge \zeta$ and consider for $\alpha<x<\beta$

$$
\begin{aligned}
p_{\alpha \beta}(x) & =P_{x}^{k_{y}}\left(\tau_{\alpha}<\tau_{\beta}\right)=P_{x}^{k_{y}}(\omega(\tau)=\alpha) \\
& =P_{x}\left(\frac{k_{y}\left(\omega_{\tau}\right)}{k_{y}(x)} ; \omega(\tau)=\alpha\right) \\
& =\frac{k_{y}(\alpha)}{k_{y}(x)} P_{x}(\omega(\tau)=\alpha) \\
& =\frac{k_{y}(\alpha)}{k_{y}(x)} \frac{s(\beta)-s(x)}{s(\beta)-s(\alpha)}
\end{aligned}
$$

in the same way

$$
p_{\beta \alpha}(x)=\frac{k_{y}(\beta)}{k_{y}(x)} \frac{s(x)-s(\alpha)}{s(\beta)-s(\alpha)}
$$

Easy calculations show that if $\beta<y$ or $\alpha>y$ then for all $u, v \in(\alpha, \beta), u<v$,

$$
p_{\alpha \beta}^{+}(u, v]=p_{\beta \alpha}^{+}(u, v]=0 .
$$

In the case $\alpha<y<\beta$ it is seen that for all $u, v$ such that $\alpha<u<y<v<\beta$ we have

$$
p^{+}(u, v]=p(y) \cdot \frac{1}{s(b)-s(y)} \quad\left(p=p_{\alpha \beta} \text { or } p_{\beta \alpha}\right) .
$$


Because this is independent of $u$ and $v$ it follows that

$$
\varkappa=\frac{1}{s(b)-s(y)} \varepsilon_{\{y\}},
$$

where $\varepsilon_{\{y\}}$ is the Dirac's measure at $y$. Hence we have (see Chapter 5.6 in [5]) the following description of $X^{y}$ : Let $T$ be an exponentially distributed random variable with mean 1 and independent of $Z$. Let

$$
A(t)=\frac{1}{s(b)-s(y)} L(t, y),
$$

and set $\zeta=\inf \{t: A(t)>T\}$. Then the process

$$
Z^{y}(t)= \begin{cases}Z(t) & \text { for } t<\zeta \\ \Delta & \text { for } t \geqq \zeta\end{cases}
$$

is identical in law with $X^{y}$.

This kind of killing with the local time is usually called elastic (compare with the construction of elastic Brownian motion in Chapter 2.3 in [5], see also [4]).

Remark 11. Notice that Proposition 4 follows now easily from Problem 2 on page 184 in [5] namely

$$
q(t ; x, y)= \begin{cases}p(t ; x, y), & \text { for } x<y, \\ p(t ; x, y) \frac{s(b)-s(y)}{s(b)-s(x)}, & \text { for } x>y,\end{cases}
$$

and, consequently,

$$
P_{x}^{k_{y}}(\zeta \in d t)=\frac{q(t ; x, y)}{s(b)-s(y)} d t
$$

ACKNOWLEDGEMENTS. I wish to thank John Walsh, who reminded me about [7], and the referee, whose suggestions much improved the paper.

\section{REFERENCES}

1. O. Barndorff-Nielsen, P. Blæsild and C. Halgreen, First hitting time models for the generalized inverse Gaussian distribution, Stochastic Processes Appl. 7 (1978), 49-54.

2. R. Duncan, Integral representation of excessive functions of a Markov process, Pacific J. Math. 39 (1971), 125-144.

3. E. B. Dynkin, Markov processes, II (Grundlehren Math. Wiss. 122) Springer-Verlag, Berlin Göttingen - Heidelberg, 1965.

4. W. Feller, Diffusion processes in one dimension, Trans. Amer. Math. Soc. 77 (1954), 1-133.

5. K. Ito and H. McKean, Diffusion processes and their sample paths (Grundlehren Math. Wiss. 125) Springer-Verlag, Berlin - Heidelberg - New York, 1965. 
6. S. Karlin and H. Taylor, A second course in stochastic processes, Academic Press, New York, 1981.

7. P. A. Meyer, R. T. Smythe, and J. B. Walsh, Birth and death of Markov processes (Proc. Sixth Berkeley Symp. on Math. Stat. and Prob.), Vol. III, pp. 295-305. Univ. of California Press, Berkeley, 1972.

8. J. Pitman and M. Yor, Bessel processes and infinity divisible laws in Stochastic Integrals (Proc. of Durham Conference 1980), ed. D. Williams, (Lecture Notes in Math. 851), SpringerVerlag, Berlin - Heidelberg - New York, 1981.

9. P. Salminen, Martin Boundaries for some space-time Markov processes, Z. Wahrsch. Verw. Gebiete 55 (1981), 41-53.

10. M. Sharpe, Some transformations of diffusions by time reversal, Ann. Probab. 8 (1980), 11571162.

11. S. Watanabe, On time inversion of one-dimensional diffusion processes, $\mathrm{Z}$. Wahr. Verw. Gebiete 31 (1975), 115-124.

12. D. Williams, Path decomposition and continuity of local time for one-dimensional diffusions, Proc. London Math. Soc. (3) 28 (1974), 738-768.

13. D. Williams, Diffusions, Markov processes, and martingales, I: Foundations, John Wiley \& Sons, Chichester - New York - Brisbane - Toronto, 1979.

\author{
MATHEMATICAL INSTITUTE \\ ABO AKADEMI \\ SF-20500 ABO 50 \\ FINLAND
}

